\title{
The Intra- and Interband Phonon-Electron Potentials in a Two-Band Model of Interacting Lattice Fermions
}

\author{
P. Tarasewicz $\mathbf{1}^{1}$
}

Received: 10 January 2015 / Accepted: 18 March 2015 / Published online: 12 April 2015

(C) The Author(s) 2015. This article is published with open access at Springerlink.com

\begin{abstract}
A new two-band polaronic Hamiltonian is derived from a generalized periodic Anderson model. The generalization consists in the completion of the periodic Anderson model with intra- and inter-band local phononelectron interactions. The derivation is achieved by making use of two canonical transformations which enable us to remove these phonon-electron potentials from the initial Hamiltonian. This decoupling procedure leads to the appearance of new terms such as a pair-exchange potential, anisotropic Kondo-lattice terms, and a density-dependent hybridization. The final Hamiltonian describes a complicated interplay between different tendencies in the investigated system. Superconductivity can compete with the Kondo effect or magnetism as well. A simplified scenario in which the effect of the single-particle hybridization on superconductivity is investigated. It is shown that this factor is very efficient as a pair-breaker.
\end{abstract}

Keywords Superconductivity · Two-band model · Phonon-electron interaction $\cdot$ Kondo lattice

\section{Introduction}

At the beginning of the high-temperature superconductivity era, the phonon-electron mechanism was not regarded

P. Tarasewicz

tarasek1@cm.umk.pl

1 Faculty of Pharmacy, Collegium Medicum in Bydgoszcz Nicolaus Copernicus University in Toruń, ul. Jagiellońska 13, 85-067 Bydgoszcz, Poland to play any relevant role in the explanation of this fascinating phenomenon. Due to the fact that undoped copper oxides were antiferromagnetically ordered Mott insulators, many authors perceived these materials as a domain being under control of purely electronic mechanisms such as for instance the spin-fluctuation one, especially due to a simple argument of the weakness of the isotope effect in them. In order to give a theoretical picture for the description of these exotic materials, some models such as the Hubbard model and the t-J one were usually invoked, e.g., in [1]. However, recent experiments have shown that this approach seems now to be incomplete. The isotope effect turns out to be quite strong even in comparison to the BCS case [2]. This takes place in underdoped regime, very close to the transition to the antiferromagnetic state. Moreover, the electromagnetic response of these systems to an external magnetic field expressed by the London penetration length manifests the isotope effect as well. There is much more evidence [2] pointing to the importance of strong phononelectron interactions and resulting polaronic phenomena in high-temperature superconductivity. The formation of the so-called pseudogap is suspected to be brought about by the strong phonon-electron interactions in contrast to the view that this is due to the opening of a spin gap [2]. Such a point of view is justified by the large isotope effect in the case of the temperature $T^{*}$ at which unpaired polarons start to bind into bipolarons. However, the bipolarons are not coherent at temperatures between the critical temperature $T_{c}$ and $T^{*}$. The coherence appears below $T_{c}$. This kind of behavior is a characteristic for systems exhibiting the so-called mixed valence, it is, for substances which contain ions of the same element differing by $2 e$ concerning their valence. Beside copper oxides, there is the multitude of them, for example, $\mathrm{Ti}_{4} \mathrm{O}_{7}, \mathrm{Na}_{x} \mathrm{~V}_{2} \mathrm{O}_{5}$ or $\mathrm{CsSbCl}_{6}$ and $\mathrm{TlF}_{2}$ which are not superconductors but 
their ground state is charge-ordered. Besides some superconductors such as $\mathrm{A15}, \mathrm{C} 15, \mathrm{~V}_{3} \mathrm{Si}, \mathrm{Nb}_{3} \mathrm{Ge}$ and cuprates such as the tungsten bronzes and the doped bismuthates ( $\mathrm{Ba}_{1-x} \mathrm{~K}_{x} \mathrm{BiO}_{3}$ and $\mathrm{BaP} b_{1-x} \mathrm{Bi}_{x} \mathrm{O}_{3}$ ) display the existence of local pairs because all of them are characterized by the poor conductivity and narrow bands being the result of the strong coupling between phonons and electrons [3]. The importance of phonon-electron interactions is also strongly stressed in [4].

In this paper, an effective model combining features of some well-known models has been derived from a generalized two-band periodic Anderson model. The two-band Anderson model was completed with two local intraband phonon-electron interactions and a local interband one describing the transfer of electrons between bands as a result of emitting or absorbing a local phonon on a lattice site. Owing to the application of two canonical transformations to the initial Hamiltonian, one has obtained the separation of fermionic and bosonic degrees of freedom. The fermionic part of the final Hamiltonian represents a system of interacting electrons dressed in the lattice deformations. As is widely known such electrons are called polarons. The resulting polarons interact to each other via local effective interand intraband Hubbard like potentials that can be either positive or negative. Apart of them, there appears a pairexchange term describing the charge transfer between both of bands. Such an interaction was derived and investigated in $[3,5-8]$ and can lead to superconductivity regardless of the sign of the aforementioned Hubbard terms. However, it is the presence of an anisotropic Kondo lattice component that is completely new. The Kondo lattice model is usually derived from the periodic Anderson model via the application of the Schrieffer-Wolff transformation as it was made in [3, 5-8]. A new Hamiltonian with the pair-exchange interaction and the Kondo lattice term was obtained from a generalized periodic Anderson model by applying the Lang-Firsov transformation and next the Schrieffer-Wolff one. However, this procedure led to the Hamiltonian without the hybridization term that was removed owing to the latter transformation. In this paper, the hybridization is still present and additionally a density-dependent hybridization appears. Moreover, the hopping of polarons takes place not only within the same bands, but also between bands. These all terms together make the final Hamiltonian be a combination of such models as the periodic Anderson model, the two-component model completed with the anisotropic Kondo lattice one and the so-called d-p model [9]. Therefore, the new Hamiltonian contains very rich physics and all of this points to the greater relevance and importance of phonon-electron interactions in new materials. One needs to add that the idea of interband phonon-electron interactions was introduced by $\mathrm{Yu}$ and Anderson in [10]. In that paper, beside the conventional hybridization between $\mathrm{s}$ and p spinless electrons, a new term describing the hybridization between those electrons assisted by phonons was admitted. Such a problem is equivalent to the single-impurity Anderson model with such two hybridization terms. This was introduced to explain some anomalies in behavior of the $A 15$ compounds, e.g., the violation of Mattheissen's rule. This idea was next undertaken in the series of papers [11-16] where the so called two-channel Kondo was investigated. One of reasons was the possibility of nonmagnetic Kondo effect in some materials, e.g., $\mathrm{SmOs}_{4} \mathrm{Sb}_{12}$ that possesses a cage-like structure.

At the end of this paper, certain simplified case has been investigated. A pair exchange potential is assumed to act between electrons from both of the bands. The potential competes with the single-electron hybridization that turns out to be a very efficient pair-breaker. Next, the results are compared to those of a case with the pairing channel introduced only in the wider of the bands.

\section{The Model and Both of Transformations}

Our starting point is the following Hamiltonian

$H=H_{0}+H_{I}$,

$$
\begin{aligned}
& H_{0}=\sum_{i \neq j, \sigma} t_{i j}^{c} c_{i \sigma}^{*} c_{j \sigma}+\left(E^{c}-\mu\right) \sum_{i \sigma} n_{i \sigma}^{c} \\
& +U^{c} \sum_{i} n_{i+}^{c} n_{i-}^{c}+\sum_{i \neq j, \sigma} t_{i j}^{d} d_{i \sigma}^{*} d_{j \sigma}+ \\
& +\left(E^{d}-\mu\right) \sum_{i \sigma} n_{i \sigma}^{d}+U^{d} \sum_{i} n_{i+}^{d} n_{i-}^{d} \\
& +V \sum_{i \sigma}\left(c_{i \sigma}^{*} d_{i \sigma}+d_{i \sigma}^{*} c_{i \sigma}\right) \\
& +U^{c d} \sum_{i \sigma \sigma^{\prime}} n_{i \sigma}^{d} n_{i \sigma^{\prime}}^{c}+\omega \sum_{i} b_{i}^{*} b_{i}
\end{aligned}
$$

$H_{I}=H_{I 1}+H_{I 2}+H_{I 3}$,

$$
\begin{aligned}
& H_{I 1}=g^{c} \sum_{i \sigma} n_{i \sigma}^{c}\left(b_{i}^{*}+b_{i}\right), H_{I 2}=g^{d} \sum_{i \sigma} n_{i \sigma}^{d}\left(b_{i}^{*}+b_{i}\right), \\
& H_{I 3}=g^{c d} \sum_{i \sigma}\left(c_{i \sigma}^{*} d_{i \sigma}+d_{i \sigma}^{*} c_{i \sigma}\right)\left(b_{i}^{*}+b_{i}\right),
\end{aligned}
$$

$c_{i \sigma}^{*}$ and $c_{i \sigma}$ represent creation and annihilation operators of a local electron from the wider s- or p-band whereas $d_{i \sigma}^{*}$ and $d_{i \sigma}$ concern local electrons from the narrower d-one, respectively. $\sigma$ denotes the spin of electrons and $i$ and $j$ refer to lattice sites. $n_{i \sigma}^{d}$ and $n_{i \sigma}^{c}$ are the number operators for electrons. $b_{i}^{*}$ and $b_{i}$ are creation and annihilation operators for a local phonon residing on site i. $t_{i j}^{c}$ and $t_{i j}^{d}$ 
denote the hopping integrals of the electrons from the $\mathrm{s}$ (p) and the $\mathrm{d}$ bands, respectively. They are equal to $-t^{c}$ and $-t^{d}$, respectively. $E^{c}$ and $E^{d}$ refer to the site energies of electrons, respectively. $V$ is the conventional on-site hybridization, and the parameters $U^{c}, U^{d}$, and $U^{c d}$ are the Coulomb interactions between electrons of the same band and the different bands, respectively. Subsequently, $\omega$ is the Einstein oscillations frequency and $\mu$ is the chemical potential. Finally, the parameters $g^{c}, g^{d}$, and $g^{c d}$ are the electron-phonon couplings. In general, $g^{c}$ and $g^{d}$ are different but for simplicity $g^{c}=g^{d}=g$ will be put here. Owing to this one avoids some difficulties with the transformation of the Hamiltonian (2.1). These difficulties will be outlined further in the text. The Hamiltonian (2.1) represents a generalized Anderson lattice model in which electrons from both of bands are strongly coupled to the same local phonons and to each other through the Coulomb interactions. $H_{I 3}$ describes the transfer of electrons between two of bands due to the absorption or the emission of local phonons. This means that an electron occupying one of orbitals on a lattice site interacts with local phonons. Due to that, the electron can stay in the same orbital or can get another one on the same site. One may say that this process is a kind of hybridization forced by the interaction of local electrons with local lattice vibrations. Both s-electrons and $\mathrm{d}$-ones are assumed to be mobile but it is possible to consider localized d-electrons. The periodic Anderson model is regarded to represent a dense magnetic alloy. A similar process was invoked in [10-16]. However, the considerations were based on the single-impurity Anderson model which represents a dilute magnetic alloy. Moreover, s, p, and localized f-electrons were investigated in [12-16]. Electrons of s-type were mixed with f-ones via the conventional hybridization while p-electrons were hybridized with f-ones owing to phonons. It should be noted that f-electrons reside on a vibrating magnetic ion and interact with mobile electrons. This process gives rise to the two-channel Kondo effect. Paper [11] considers the possibility of hybridization of conduction electrons of one type with electrons localized on such an impurity as a mechanism of tunneling in molecular junctions. The important fact is that in all of those papers the electron-phonon terms were not eliminated by means of the appropriate unitary transformation.

It is convenient to transform the initial Hamiltonian to a new one expressed in terms of small polarons similarly as it was made ,e.g., in [2, 3]. To this end, let us use a LangFirsov type transformation

$$
\begin{aligned}
\tilde{H} & =U_{1} H U_{1}^{\star}, U_{1}=e^{S_{1}}, \\
S_{1} & :=\frac{g}{\omega} \sum_{i \sigma}\left(n_{i \sigma}^{c}+n_{i \sigma}^{d}\right)\left(b_{i}^{*}-b_{i}\right) .
\end{aligned}
$$

It yields

$$
\begin{aligned}
& \tilde{H}=\sum_{i \neq j, \sigma} t_{i j}^{c} c_{i \sigma}^{*} c_{j \sigma} e^{\frac{g}{\omega}\left(b_{i}^{*}-b_{i}-b_{j}^{*}+b_{j}\right)} \\
& +\sum_{i \neq j, \sigma} t_{i j}^{d} d_{i \sigma}^{*} d_{j \sigma} e^{\frac{g}{\omega}\left(b_{i}^{*}-b_{i}-b_{j}^{*}+b_{j}\right)}+ \\
& +\tilde{E}^{c} \sum_{i \sigma} n_{i \sigma}^{c}+\tilde{U}^{c} \sum_{i} n_{i+}^{c} n_{i-}^{c}+\tilde{E}^{d} \sum_{i \sigma} n_{i \sigma}^{d} \\
& +\tilde{U}^{d} \sum_{i} n_{i+}^{d} n_{i-}^{d} \\
& +\tilde{V} \sum_{i \sigma}\left(c_{i \sigma}^{*} d_{i \sigma}+d_{i \sigma}^{*} c_{i \sigma}\right)+ \\
& -\frac{2 g g^{c d}}{\omega} \sum_{i}\left[\left(n_{i+}^{c}+n_{i+}^{d}\right)\right. \\
& \left.\times\left(c_{i-}^{*} d_{i-}+d_{i-}^{*} c_{i-}\right)+\left(n_{i-}^{c}+n_{i-}^{d}\right)\left(c_{i+}^{*} d_{i+}+d_{i+}^{*} c_{i+}\right)\right]+ \\
& +\tilde{U}^{c d} \sum_{i \sigma \sigma^{\prime}} n_{i \sigma}^{d} n_{i \sigma^{\prime}}^{c}+\omega \sum_{i} b_{i}^{*} b_{i} \\
& +g^{c d} \sum_{i \sigma}\left(c_{i \sigma}^{*} d_{i \sigma}+d_{i \sigma}^{*} c_{i \sigma}\right)\left(b_{i}^{*}+b_{i}\right),
\end{aligned}
$$

with the definitions of some new symbols in the Hamiltonian above

$$
\begin{aligned}
& \tilde{E}^{c}:=E^{c}-\mu-\frac{g^{2}}{\omega}, \tilde{E}^{d}:=E^{d}-\mu-\frac{g^{2}}{\omega}, \tilde{U}^{c}:=U^{c}-\frac{2 g^{2}}{\omega}, \\
& \tilde{U}^{c d}:=U^{c d}-\frac{2 g^{2}}{\omega}, \tilde{V}:=V-2 \frac{g g^{c d}}{\omega}, \tilde{U}^{d}:=U^{d}-\frac{2 g^{2}}{\omega} .
\end{aligned}
$$

The transformed creation and anihilation operators for bosons and fermions are given below

$$
\begin{aligned}
& \tilde{c}_{i \sigma}^{*}=c_{i \sigma}^{*} e^{\frac{g}{\omega}\left(b_{i}^{*}-b_{i}\right)}, \tilde{c}_{i \sigma}=c_{i \sigma} e^{-\frac{g}{\omega}\left(b_{i}^{*}-b_{i}\right)}, \\
& \tilde{d}_{i \sigma}^{*}=d_{i \sigma}^{*} e^{\frac{g}{\omega}\left(b_{i}^{*}-b_{i}\right)}, \tilde{d}_{i \sigma}=d_{i \sigma} e^{-\frac{g}{\omega}\left(b_{i}^{*}-b_{i}\right)}, \\
& \tilde{b}_{i}^{*}=b_{i}^{*}-\frac{g}{\omega}\left(n_{i+}^{c}+n_{i-}^{c}+n_{i+}^{d}+n_{i-}^{d}\right), \\
& \tilde{b}_{i}=b_{i}-\frac{g}{\omega}\left(n_{i+}^{c}+n_{i-}^{c}+n_{i+}^{d}+n_{i-}^{d}\right) .
\end{aligned}
$$

As a result, one obtains a Hamiltonian that has the modified coupling constants of all the electron-electron interactions and also both the hopping parameters and the site-energies. Depending on the strength of the intraband and interband Coulomb repulsions as well as the phonon-electron interactions, the effective polaron-polaron potentials can be repulsive or attractive ones. As is widely known attractive interactions between polarons lead to superconductivity. Moreover, new terms appear in the resulting Hamiltonian. They describe a kind of the hybridization which is densitydependent, i.e., they contribute if a site is occupied by a polaron with opposite spin. This is a result of the presence of $H_{I 3}$ term in the initial Hamiltonian which has not been 
eliminated by making use of the transformation $U_{1}$. In order to eliminate one, there is a need to apply another unitary transformation to the transformed Hamiltonian. This new transformation reads

$\bar{H}_{F}=U_{2} \tilde{H} U_{2}^{\star}, \quad U_{2}=e^{S_{2}}$,

where

$S_{2}:=\frac{g^{c d}}{\omega} \sum_{i \sigma}\left(c_{i \sigma}^{*} d_{i \sigma}+d_{i \sigma}^{*} c_{i \sigma}\right)\left(b_{i}^{*}-b_{i}\right)$.

One remark should be made here, namely the equality $g^{c}=$ $g^{d}$ entails the removal of another difficulty. In general, for $g^{c} \neq g^{d}\left[S_{1}, S_{2}\right]=0$ does not hold. In such a case, due to $\left[S_{1}, S_{2}\right] \neq 0$, the Hamiltonian $U_{1} U_{2} H U_{2}^{\star} U_{1}^{\star}$ is not the same as the Hamiltonian $U_{2} U_{1} H U_{1}^{\star} U_{2}^{\star}$ and in order to make amendments for this there is the necessity to incorporate the known formula $e^{(A+B)}=e^{A} e^{B} e^{-\frac{1}{2}[A, B]}$. Unfortunately, this leads to significant complications in finding the new outcome. That is why it is convenient to put $g^{c}=g^{d}$ at the cost of some loss of the generality. Owing to this, $\left[S_{1}, S_{2}\right]=0$ is satisfied and the order of the transformations applied to the Hamiltonian (2.1) is arbitrary. Now, let us transform this Hamiltonian by making use of the transformation $U_{2}$. The transformed operators take the following form

$$
\begin{aligned}
\bar{b}_{i}^{*} & =b_{i}^{*}-\frac{g^{c d}}{\omega} \sum_{i \sigma}\left(c_{i \sigma}^{*} d_{i \sigma}+d_{i \sigma}^{*} c_{i \sigma}\right), \\
\bar{b}_{i} & =b_{i}-\frac{g^{c d}}{\omega} \sum_{i \sigma}\left(c_{i \sigma}^{*} d_{i \sigma}+d_{i \sigma}^{*} c_{i \sigma}\right), \\
\bar{c}_{i \sigma}^{*} & =c_{i \sigma}^{*} \cosh \left[\frac{g^{c d}}{\omega}\left(b_{i}^{*}-b_{i}\right)\right]+d_{i \sigma}^{*} \sinh \left[\frac{g^{c d}}{\omega}\left(b_{i}^{*}-b_{i}\right)\right], \\
\bar{c}_{i \sigma} & =c_{i \sigma} \cosh \left[\frac{g^{c d}}{\omega}\left(b_{i}^{*}-b_{i}\right)\right]-d_{i \sigma} \sinh \left[\frac{g^{c d}}{\omega}\left(b_{i}^{*}-b_{i}\right)\right], \\
\bar{d}_{i \sigma}^{*} & =d_{i \sigma}^{*} \cosh \left[\frac{g^{c d}}{\omega}\left(b_{i}^{*}-b_{i}\right)\right]+c_{i \sigma}^{*} \sinh \left[\frac{g^{c d}}{\omega}\left(b_{i}^{*}-b_{i}\right)\right], \\
\bar{d}_{i \sigma} & =d_{i \sigma} \cosh \left[\frac{g^{c d}}{\omega}\left(b_{i}^{*}-b_{i}\right)\right]-c_{i \sigma} \sinh \left[\frac{g^{c d}}{\omega}\left(b_{i}^{*}-b_{i}\right)\right] .
\end{aligned}
$$

Before the new Hamiltonian is given certain thing should be made here. This Hamiltonian is to have the purely fermionic character. That is why we would like to get rid of bosonic contributions from it. The Hamiltonian $\bar{H}_{F}$ has terms modified by some functional factors involving the lattice deformation. To deal with these terms, the Hamiltonian $\bar{H}_{F}$ is averaged over the unperturbed phonon eigenstates of $H_{p h}=\omega \sum_{i} b_{i}^{*} b_{i}$ (see Appendix) and the energy of phonons is neglected in analogy to that done in [3]. Therefore, let us notice that

$\operatorname{Tr}\left(\rho e^{ \pm \alpha\left(b_{i}^{*}-b_{i}\right)}\right)=e^{-\frac{1}{2} \alpha^{2} \operatorname{coth} \frac{\beta \omega}{2}} \approx e^{-\frac{1}{2} \alpha^{2}}$, where $\beta=\frac{1}{k T}, \alpha$ is an arbitrary constant and at low temperatures $\operatorname{coth}\left(\frac{\beta \omega}{2}\right) \approx 1$ while

$\rho=\frac{e^{-\beta H_{p h}}}{\operatorname{Tr} e^{-\beta H_{p h}}}$.

The full transformed Hamiltonian $\bar{H}_{F}$ for the arbitrary nonzero $g^{c d}$ together with the aforementioned factors after averaging over $\rho$ have been relegated to Appendix.

The resulting Hamiltonian in the $\frac{g^{c d}}{\omega} \gg 1$ limit takes the following form

$$
\begin{aligned}
\bar{H}= & \sum_{i \neq j, \sigma} \bar{t}_{i j}^{c} c_{i \sigma}^{*} c_{j \sigma}+\sum_{i \neq j, \sigma} \bar{t}_{i j}^{d} d_{i \sigma}^{*} d_{j \sigma} \\
& +\sum_{i \neq j, \sigma} \bar{t}_{i j}^{c d}\left(c_{i \sigma}^{*} d_{j \sigma}+d_{i \sigma}^{*} c_{j \sigma}\right) \\
& +\bar{E}^{c d} \sum_{i \sigma}\left(n_{i \sigma}^{c}+n_{i \sigma}^{d}\right)+ \\
+ & \bar{U}_{c c}^{d d} \sum_{i}\left(n_{i+}^{c} n_{i-}^{c}+n_{i+}^{d} n_{i-}^{d}\right) \\
+ & \bar{U}_{c d}^{d c} \sum_{i}\left(n_{i+}^{c} n_{i-}^{d}+n_{i+}^{d} n_{i-}^{c}\right) \\
+ & \bar{V} \sum_{i \sigma}\left(c_{i \sigma}^{*} d_{i \sigma}+d_{i \sigma}^{*} c_{i \sigma}\right)+ \\
& -\frac{2 g g^{c d}}{\omega} \sum_{i}\left[\left(n_{i+}^{c}+n_{i+}^{d}\right)\right. \\
& \left.\left(c_{i-}^{*} d_{i-}+d_{i-}^{*} c_{i-}\right)+\left(n_{i-}^{c}+n_{i-}^{d}\right)\left(c_{i+}^{*} d_{i+}+d_{i+}^{*} c_{i+}\right)\right]+ \\
+ & \bar{V}_{p} \sum_{i}\left(c_{i+}^{*} c_{i-}^{*} d_{i-} d_{i+}+d_{i+}^{*} d_{i-}^{*} c_{i-} c_{i+}\right) \\
+ & \bar{J}^{ \pm} \sum_{i}\left(S_{i}^{c+} S_{i}^{d-}+S_{i}^{d+} S_{i}^{c-}\right)+\bar{J}^{z} \sum_{i} S_{i}^{c z} S_{i}^{d z},
\end{aligned}
$$

where the effective parameters are as follows

$$
\begin{aligned}
& \bar{t}^{c}=-\frac{1}{4}\left(t^{c}-t^{d}\right) e^{-\frac{\left(g-g^{c d}\right)^{2}}{\omega^{2}}}, \bar{t}^{d}=\frac{1}{4}\left(t^{c}-t^{d}\right) e^{\left.-\frac{(g-g c d}{\omega^{2}}\right)^{2}}, \\
& \bar{t}^{c d}=\frac{1}{4}\left(t^{c}+t^{d}\right) e^{-\frac{\left(g-g^{c d}\right)^{2}}{\omega^{2}}}, \\
& \bar{U}_{c c}^{d d}=\frac{3}{8}\left(U^{c}+U^{d}\right)+\frac{1}{4} U^{c d}-2 \frac{g^{2}}{\omega}, \\
& \bar{U}_{c d}^{d c}=\frac{1}{8}\left(U^{c}+U^{d}\right)+\frac{3}{4} U^{c d}+2 \frac{g^{c d^{2}}-g^{2}}{\omega},
\end{aligned}
$$




$$
\begin{aligned}
& \bar{E}^{c}= \frac{1}{2}\left(E^{c}+E^{d}+U^{c d}\right)-\mu-\frac{2 g^{2}+g^{c d}}{\omega}, \\
& \bar{V}_{p}=-\frac{1}{8}\left(U^{c}+U^{d}\right)+\frac{1}{4} U^{c d}-2 \frac{g^{c d^{2}}}{\omega}, \\
& \bar{V}=V-2 \frac{g g^{c d}}{\omega}, \quad \bar{J}^{ \pm}=-\frac{1}{8}\left(U^{c}+U^{d}\right)+\frac{1}{4} U^{c d} \\
& \quad+2 \frac{g^{c d^{2}}}{\omega}, \\
& \bar{J}^{z}=8 \frac{g^{c d^{2}}}{\omega} .
\end{aligned}
$$

The spin operators are defined as follows: $S_{i}^{c z}=\frac{1}{2}\left(n_{i+}^{c}-\right.$ $\left.n_{i-}^{c}\right), S_{i}^{d z}=\frac{1}{2}\left(n_{i+}^{d}-n_{i-}^{d}\right), S_{i}^{c+}=c_{i+}^{*} c_{i-}, S_{i}^{c-}=c_{i-}^{*} c_{i+}$, $S_{i}^{d+}=d_{i+}^{*} d_{i-}$ and $S_{i}^{d-}=d_{i-}^{*} d_{i+}$. The hopping integrals were assumed to be $\bar{t}_{i j}^{c}=\bar{t}^{c}$ and $\bar{t}_{i j}^{d}=\bar{t}^{d} \cdot \bar{t}_{i j}^{c d}$ is determined by those ones. The effective Hamiltonian (2.8) describes a many-polaron system in which some new terms appear. A new hopping term describes the transfer of polarons of different bands between adjacent sites. Additionally, new interactions are found as well. Beside the effective local Coulomb interactions acting between polarons with opposite spins a pair-exchange potential appears. This kind of potential was derived from a two-band periodic Anderson model via the application of the Lang-Firsov transformation and next the Schrieffer-Wolff transformation [3]. This interaction can lead to superconductivity on its own. Moreover, spin-spin interactions are present as well. They represent a component that can be recognized as an anisotropic Kondo lattice model. Therefore, the Hamiltonian $\bar{H}$ describes a many-polaron system in which at least three ordering tendencies compete with each other, it is, superconductivity, the Kondo effect and magnetism that can be driven by RKKY interactions between neighboring local moments mediated by conduction electrons $[17,18]$.

It could be interesting to give a short analysis of the underlying physics. Let us assume that $g^{c d} \approx g$ and $\frac{g}{\omega} \approx$ $\frac{g^{c d}}{\omega} \gg 1$ hold, then note that $\bar{t}^{c}=-\frac{1}{4}\left(t^{c}-t^{d}\right), \bar{t}^{d}=$ $\frac{1}{4}\left(t^{c}-t^{d}\right), \bar{t}^{c d}=\frac{1}{4}\left(t^{c}+t^{d}\right)$. Moreover, let us admit the choice $U^{c} \approx U^{d} \approx U^{c d}=U$. This leads to the following values of the coupling constants

$$
\begin{aligned}
& \bar{U}_{c c}^{d d} \approx U-2 \frac{g^{2}}{\omega}, \quad \bar{U}_{c d}^{d c} \approx U, \quad \bar{V} \approx V-2 \frac{g^{2}}{\omega}, \\
& \bar{V}_{p} \approx-2 \frac{g^{2}}{\omega}, \quad \bar{J}^{ \pm} \approx 2 \frac{g^{2}}{\omega}, \quad \bar{J}^{z} \approx 8 \frac{g^{2}}{\omega} .
\end{aligned}
$$

At once, one can recognize the anisotropic Kondo lattice component with antiferromagnetic coupling constants. It is clear that in this scenario there can be two attractive potentials leading to superconductivity which compete with tendencies driven by the Kondo lattice term. In other words, in both of the bands, local bipolarons are formed. At sufficiently low temperatures, they condense and as a result superconductivity appears. This process is strengthened by the presence of the pair-exchange term. In general, the Kondo lattice term has the tendency to break pairs and hamper the occurrence of superconductivity, although there are papers, e.g., [19], reporting the possibility of the appearance of superconductivity in the Kondo lattice model.

Of course, some different cases are possible. If for instance $U$ is sufficiently large, then $\bar{U}_{c c}^{d d}>0$ and $\bar{U}_{c d}^{d c}>0$ and only one mechanism responsible for superconductivity remains, namely that one driven by the pair-exchange potential.

It is worth noticing that the single-particle hybridization is still present in the transformed Hamiltonian. This term is usually removed from both the single-impurity Anderson Hamiltonian and the periodic Anderson Hamiltonian by application of the appropriate Schrieffer-Wolff transformation to them $[3,20]$. As a result, one obtains the Kondo model and the Kondo lattice model, respectively. Here, the usage of the canonical transformations (2.5) and (2.7) not only results in the eliciting of the Kondo-lattice component, but also keeps the hybridization as well. A completely new fact is that the Kondo-lattice term is obtained by removing the phonon-electron interactions from the initial Hamiltonian. Physically, it means that the processes leading to the states occupied by polarons from the different bands and with opposite spins will be stronger and superconductivity in both bands will be easier suppressed.

\section{The Excitation Spectrum of a Simplified Version}

In this section and the next one, a simplified scenario will be investigated. This is represented by the following Hamiltonian:

$$
\begin{aligned}
& H_{\text {red }}=\sum_{i \neq j, \sigma} t_{i j}^{c} c_{i \sigma}^{*} c_{j \sigma}+\sum_{i \neq j, \sigma} t_{i j}^{d} d_{i \sigma}^{*} d_{j \sigma} \\
& +\left(E^{d}-\mu\right) \sum_{i \sigma} n_{i \sigma}^{d}+\left(E^{c}-\mu\right) \sum_{i \sigma} n_{i \sigma}^{c}+ \\
& +V \sum_{i \sigma}\left(c_{i \sigma}^{*} d_{i \sigma}+d_{i \sigma}^{*} c_{i \sigma}\right) \\
& +V_{p} \sum_{i}\left(c_{i+}^{*} c_{i-}^{*} d_{i-} d_{i+}+d_{i+}^{*} d_{i-}^{*} c_{i-} c_{i+}\right)
\end{aligned}
$$

The Hamiltonian (3.1) describes a two-band fermion system in which the exchange of both single particles, and their pairs between bands is admitted. The idea of the exchange of electron pairs between two bands as a possible 
mechanism of superconductivity was introduced at the same time by Moskalenko [21] and Suhl et al. [22]. Here, the single-particle hybridization is taken into account as a pair breaker. In systems with narrow bands Coulomb repulsions are frequently regarded to be destructive to Cooper pairs. However, in this paper, we would like to focus our attention to hybridization as a factor destabilizing superconductivity. This mechanism can take place in heavy-fermion superconductivity. The Hamiltonian (3.1) does not include the spinspin interactions and the interband hopping. The Coulomb terms have been neglected as well. The density-dependent hybridization is assumed to be incorporated into the effective single-particle hybridization.

The Hamiltonian (3.1) transformed to the momentum space via $c_{i \sigma}^{*}=\frac{1}{\sqrt{L}} \sum_{\mathbf{k}} e^{-i \mathbf{k} \mathbf{R}_{i}} c_{\mathbf{k} \sigma}^{*}$ and $d_{i \sigma}^{*}=\frac{1}{\sqrt{L}} \sum_{\mathbf{k}} e^{-i \mathbf{k} \mathbf{R}_{i}} d_{\mathbf{k} \sigma}^{*}$, where $L$ denotes the number of lattice sites and the vector $\mathbf{R}_{i}$ stands for the position of the $i$-th site, and approximated by the mean field treatment, reads

$$
\begin{aligned}
& H_{M F}=\sum_{\mathbf{k}}\left(\xi_{\mathbf{k}}^{c}\left(n_{\mathbf{k}+}^{c}+n_{-\mathbf{k}-}^{c}\right)+\xi_{\mathbf{k}}^{d}\left(n_{\mathbf{k}+}^{d}+n_{-\mathbf{k}-}^{d}\right)\right. \\
& +V\left(c_{\mathbf{k}+}^{*} d_{\mathbf{k}+}+d_{\mathbf{k}+}^{*} c_{\mathbf{k}+}+c_{-\mathbf{k}-}^{*} d_{-\mathbf{k}-}\right. \\
& \left.+d_{-\mathbf{k}-}^{*} c_{-\mathbf{k}-}\right)+ \\
& \left.-\Delta^{d}\left(c_{\mathbf{k}+}^{*} c_{-\mathbf{k}-}^{*}+c_{-\mathbf{k}-} c_{\mathbf{k}+}\right)-\Delta^{c}\left(d_{\mathbf{k}+}^{*} d_{-\mathbf{k}-}^{*}+d_{-\mathbf{k}-} d_{\mathbf{k}+}\right)\right) \\
& +2 L \frac{\Delta^{c} \Delta^{d}}{V_{p}}=\sum_{\mathbf{k}} H_{\mathbf{k} M F}+2 L \frac{\Delta^{c} \Delta^{d}}{V_{p}},
\end{aligned}
$$

with the gap parameters

$$
\Delta^{c}=\frac{V_{p}}{L} \sum_{\mathbf{k}}\left\langle c_{\mathbf{k}+}^{*} c_{-\mathbf{k}-}^{*}\right\rangle_{H_{M F}}=\frac{V_{p}}{L} \sum_{\mathbf{k}}\left\langle c_{-\mathbf{k}-} c_{\mathbf{k}+}\right\rangle_{H_{M F}},
$$

$$
\Delta^{d}=\frac{V_{p}}{L} \sum_{\mathbf{k}}\left\langle d_{\mathbf{k}+}^{*} d_{-\mathbf{k}-}^{*}\right\rangle_{H_{M F}}=\frac{V_{p}}{L} \sum_{\mathbf{k}}\left\langle d_{-\mathbf{k}-} d_{\mathbf{k}+}\right\rangle_{H_{M F}}
$$

The average $\langle A\rangle_{H_{M F}}=\frac{\operatorname{Tr} A e^{-\beta H_{M F}}}{\operatorname{Tr} e^{-\beta H_{M F}}}$, where $A$ is an arbitrary operator. $\xi_{\mathbf{k}}^{c}=\varepsilon_{\mathbf{k}}^{c}+E^{c}-\mu, \xi_{\mathbf{k}}^{d}=\varepsilon_{\mathbf{k}}^{d}+E^{d}-\mu$ with dispersion relations for both of bands $\varepsilon_{\mathbf{k}}^{c}$ and $\varepsilon_{\mathbf{k}}^{d}$. We assume the two-dimensional square lattice with the nearestneighbor hopping.
Now, one can diagonalize the Hamiltonian $H_{\mathbf{k} M F}$. The Hamiltonian $H_{\mathbf{k} M F}$ acts in the 16-dimensional space $\mathcal{M}_{\mathbf{k}}$ spanned by the vectors $\left|n_{1} n_{2} m_{1} m_{2}\right\rangle_{\mathbf{k}}=\left(c_{\mathbf{k}+}^{*}\right)^{n_{1}}\left(c_{-\mathbf{k}-}^{*}\right)^{n_{2}}$ $\left(d_{\mathbf{k}+}^{*}\right)^{m_{1}}\left(d_{-\mathbf{k}-}^{*}\right)^{m_{2}}|0\rangle$, where $n_{i}=0,1$ and $m_{i}=0,1$ for $i=1,2$. The diagonalization of this Hamiltonian leads to the problem of the diagonalization of a $16 \times 16$ matrix and thus a formidable task. However, one can apply the method resembling that one of Czerwonko $[23,24]$ to the Hamiltonian if one defines the operators $\Lambda_{\mathbf{k}}=\Lambda_{\mathbf{k}}^{c}+\Lambda_{\mathbf{k}}^{d}, \Lambda_{\mathbf{k}}^{c}=$ $n_{\mathbf{k}+}^{c}-n_{-\mathbf{k}-}^{c}$ and $\Lambda_{\mathbf{k}}^{d}=n_{\mathbf{k}+}^{d}-n_{-\mathbf{k}-}^{d}$ then the following commutation relations are satisfied

$\left[\Lambda_{\mathbf{k}}^{c}, H_{\mathbf{k} M F}\right]=\left[\Lambda_{\mathbf{k}}^{d}, H_{\mathbf{k} M F}\right]=\left[\Lambda_{\mathbf{k}}, H_{\mathbf{k} M F}\right]=0$.

This fact enables us to simplify the problem to the diagonalization of the Hamiltonian in invariant subspaces of $\mathcal{M}_{\mathbf{k}}$. Ultimately, the structure of the $\mathcal{M}_{\mathbf{k}}$ is as follows (the subscript $\mathbf{k}$ will be suppressed in the sequel):

(A) There are two 1-dimensional common subspaces $\mathcal{M}_{i}$ $(i=1,2)$ of the operator $\Lambda$ i $H_{M F}$. They are spanned, respectively, by the following two vectors with the corresponding eigenvalues $\lambda$ and $E$ of this operators equal as follows:

$$
\begin{aligned}
& \text { 1. }|1010\rangle \lambda=2 E_{1}=\xi^{c}+\xi^{d} \\
& \text { 2. }|0101\rangle \lambda=-2 E_{2}=\xi^{c}+\xi^{d}
\end{aligned}
$$

(B) There are also two 4-dimensional common subspaces $\mathcal{M}_{i}(i=3,4)$ of $\Lambda$ and $H_{M F}$ spanned by the following quartets of vectors $\left|n_{1} n_{2} m_{1} m_{2}\right\rangle$ with eigenvalues of these operators equal as follows:

$$
\begin{aligned}
& |1000\rangle|0010\rangle|1110\rangle|1011\rangle \lambda=1 E_{3}^{j} j= \\
& 1,2,3,4 \\
& \text { 4. } \begin{array}{l}
|0100\rangle|0001\rangle|1101\rangle|0111\rangle \lambda=-1 E_{4}^{j} j= \\
1,2,3,4
\end{array}
\end{aligned}
$$

where

$$
\begin{aligned}
& E_{3}^{1}=E_{4}^{1}=\xi^{c}+\xi^{d}-\frac{\sqrt{2}}{2} E_{m}, \quad E_{3}^{2}=E_{4}^{2}=\xi^{c}+\xi^{d}+\frac{\sqrt{2}}{2} E_{m}, \\
& E_{3}^{3}=E_{4}^{3}=\xi^{c}+\xi^{d}-\frac{\sqrt{2}}{2} E_{n}, \quad E_{3}^{4}=E_{4}^{4}=\xi^{c}+\xi^{d}+\frac{\sqrt{2}}{2} E_{n}
\end{aligned}
$$

$$
\begin{aligned}
& E_{m}=\sqrt{\xi^{c^{2}}+\xi^{d^{2}}+\Delta^{c^{2}}+\Delta^{d^{2}}+2 V^{2}-E_{i n}} \\
& E_{n}=\sqrt{\xi^{c^{2}}+\xi^{d^{2}}+\Delta^{c^{2}}+\Delta^{d^{2}}+2 V^{2}+E_{i n}}
\end{aligned}
$$

$E_{i n}=\frac{\sqrt{\xi^{c 4}+\xi^{d^{4}}+\Delta^{d^{4}}+\Delta^{c 4}-2 \xi^{c 2} \xi^{d^{2}}-2 \xi^{c^{2}} \Delta^{c 2}+2 \xi^{d^{2}} \Delta^{c^{2}}+2 \xi^{c^{2}} \Delta^{d^{2}}-2 \xi^{d^{2}} \Delta^{d^{2}}+}}{-2 \Delta^{c^{2}} \Delta^{d^{2}}+4 \xi^{c^{2}} V^{2}+4 \xi^{d^{2}} V^{2}+8 \xi^{c} \xi^{d} V^{2}+4 \Delta^{c^{2}} V^{2}+4 \Delta^{d^{2}} V^{2}-8 \Delta^{c} \Delta^{d} V^{2}}$. 
The eigenvectors of $H_{M F}$ in these subspaces have the form

$\left|E_{3}^{j}\right\rangle=a_{3}^{j}|1000\rangle+b_{3}^{j}|0010\rangle+c_{3}^{j}|1110\rangle+d_{3}^{j}|1011\rangle$

and

$\left|E_{4}^{j}\right\rangle=a_{4}^{j}|0100\rangle+b_{4}^{j}|0001\rangle+c_{4}^{j}|1101\rangle+d_{4}^{j}|0111\rangle$.

The components are the same for these subspaces and take the following form

$$
\begin{aligned}
a_{3 / 4}= & \frac{c_{3 / 4}^{j}}{\xi^{c}-E_{3 / 4}^{j}}\left[\Delta^{c}-V R_{3 / 4}^{j}\right], \quad b_{3 / 4}^{j} \\
= & c_{3 / 4}^{j} R_{3 / 4}^{j}, \\
& d_{3 / 4}^{j}=\frac{c_{3 / 4}^{j}}{2 \xi^{c}+\xi^{d}-E_{3 / 4}^{j}}\left[V+\Delta^{d} R_{3 / 4}^{j}\right]
\end{aligned}
$$

with

$$
\begin{aligned}
R_{3 / 4}^{j} & = \\
& =\frac{\left(\Delta^{c}\left(V-\Delta^{c}\right)+\left(2 \xi^{d}+\xi^{c}-E_{3 / 4}^{j}\right)\left(\xi^{c}-E_{3 / 4}^{j}\right)\right)\left(2 \xi^{c}+\xi^{d}-E_{3 / 4}^{j}\right)-V\left(\xi^{c}-E_{3 / 4}^{j}\right)\left(V+\Delta^{d}\right)}{\left(V\left(V-\Delta^{c}\right)-\left(\xi^{d}-E_{3 / 4}^{j}\right)\left(\xi^{c}-E_{3 / 4}^{j}\right)\right)\left(2 \xi^{c}+\xi^{d}-E_{3 / 4}^{j}\right)+\Delta^{d}\left(\Delta^{d}+V\right)\left(\xi^{c}-E_{3 / 4}^{j}\right)} .
\end{aligned}
$$

The component $c_{3 / 4}^{j}$ can be found from the normalization condition:

$a_{3 / 4}^{j}{ }^{2}+b_{3 / 4}^{j}{ }^{2}+c_{3 / 4}^{j}{ }^{2}+d_{3 / 4}^{j}{ }^{2}=1$.

(C) There is one 6-dimensional common subspace $\mathcal{M}_{5}$ of $\Lambda$ and $H_{M F}$ spanned by the vectors $|0000\rangle,|1100\rangle$, $|0011\rangle,|1001\rangle,|0110\rangle,|1111\rangle$. The eigenvalue of $\Lambda$ in $\mathcal{M}_{5}$ is $\lambda=0$, whereas the Hamiltonian has the following eigenvalues:

$$
E_{5}^{1}=E_{5}^{2}=\xi^{c}+\xi^{d}, \quad E_{5}^{3}=\xi^{c}+\xi^{d}-E_{o 1},
$$

$$
\begin{aligned}
& E_{5}^{4}=\xi^{c}+\xi^{d}+E_{o 1}, \quad E_{5}^{5}=\xi^{c}+\xi^{d}-E_{o 2}, \\
& E_{5}^{6}=\xi^{c}+\xi^{d}+E_{o 2},
\end{aligned}
$$

where

$E_{o 1}=\sqrt{\xi^{c^{2}}+\xi^{d^{2}}+\Delta^{d^{2}}+\Delta^{c^{2}}+2 V^{2}+2 \bar{E}_{i n}}$,

$E_{o 2}=\sqrt{\xi^{c^{2}}+\xi^{d^{2}}+\Delta^{d^{2}}+\Delta^{c^{2}}+2 V^{2}-2 \bar{E}_{i n}}$ and

$\bar{E}_{i n}=\sqrt{\xi^{c 2} \xi^{d^{2}}+\Delta^{c 2} \xi^{c 2}+\Delta^{d^{2}} \xi^{d^{2}}+\Delta^{c 2} \Delta^{d^{2}}-2 \xi^{c} \xi^{d} V^{2}+2 \Delta^{c} \Delta^{d} V^{2}+V^{4}}$.

The eigenvectors in this subspace for $i=1,2,3,4,5$,

6 are given by:

$$
\begin{aligned}
\left|E_{5}^{i}\right\rangle= & a_{5}^{i}|0000\rangle+b_{5}^{i}|1100\rangle+c_{5}^{i}|0011\rangle+d_{5}^{i}|0110\rangle \\
& +e_{5}^{i}|1001\rangle+f_{5}^{i}|1111\rangle,
\end{aligned}
$$

where the components take the following form

$$
a_{5}^{i}=\frac{c_{5}^{i}}{E_{5}^{i}}\left[\Delta^{c}-\Delta^{d} R_{5}^{i}\right], \quad b_{5}^{i}=-c_{5}^{i} R_{5}^{i},
$$

$$
\begin{gathered}
d_{5}^{i}=-e_{5}^{i}=-\frac{V c_{5}^{i}}{\xi^{c}+\xi^{d}-E_{5}^{i}}\left[1-R_{5}^{i}\right], \\
f_{5}^{i}=\frac{c_{5}^{i}}{2 \xi^{c}+2 \xi^{d}-E_{5}^{i}}\left[\Delta^{d}-\Delta^{c} R_{5}^{i}\right]
\end{gathered}
$$

with

$R_{5}^{i}=\frac{\left(2 \xi^{c}+2 \xi^{d}-E_{5}^{i}\right)\left(\Delta^{c}\left(\Delta^{c}-\Delta^{d}\right)-\left(2 \xi^{d}-E_{5}^{i}\right) E_{5}^{i}\right)+E_{5}^{i}\left(\Delta^{d}-\Delta^{c}\right) \Delta^{d}}{\left(2 \xi^{c}+2 \xi^{d}-E_{5}^{i}\right)\left(\Delta^{d}\left(\Delta^{c}-\Delta^{d}\right)+\left(2 \xi^{c}-E_{5}^{i}\right) E_{5}^{i}\right)+E_{5}^{i}\left(\Delta^{d}-\Delta^{c}\right) \Delta^{c}}$. 
The component $c_{5}^{i}$ can be found from the normalization condition:

$$
a_{5}^{i^{2}}+{b_{5}^{i^{2}}}^{2}+{c_{5}^{i^{2}}}^{2}+d_{5}^{i^{2}}+e_{5}^{i^{2}}+{f_{5}^{i^{2}}}^{2}=1
$$

The spectrum is complicated but one can recognize the ground state energy and the corresponding vector relatively easily. The lowest energy state vector belongs to the subspace $\mathcal{M}_{\mathbf{k} 5}$ and its number is $i=3$. Therefore, the ground state energy is $E_{\mathbf{k} 5}^{3}$. This vector has a much more complicated form than the conventional BCS ground state vector. As one can see, the structure of the ground state reveals some interesting features since it incorporates states occupied by pairs of electrons from the same band, the quartet state occupied by two types of pairs as well as the Kondotype states with single c-electron and d-electron of opposite spins.

\section{The Zero-Temperature Gaps and the Critical Temperature}

Now, after finding the solution of the eigenproblem of Hamiltonian $H_{M F}$, one can look at the gap parameters $\Delta^{c}$ and $\Delta^{d}$ as functions of hybridization $V$ at zero temperature. In order to determine them, one can use the equations (3.3) and (3.4) or resort to the following conditions:

$L^{-1} \frac{\partial E_{G}}{\partial \Delta^{c}}=0 \quad$ and $\quad L^{-1} \frac{\partial E_{G}}{\partial \Delta^{d}}=0$,

where $E_{G}=\sum_{\mathbf{k}} \xi_{\mathbf{k}}^{c}+\sum_{\mathbf{k}} \xi_{\mathbf{k}}^{d}-\sum_{\mathbf{k}} E_{\mathbf{k} o 1}+2 L \frac{\Delta^{c} \Delta^{d}}{V_{p}}$ is the ground state energy. One obtains

$$
\begin{aligned}
\Delta^{d}= & \frac{V_{p} \Delta^{c}}{2 D e} \int_{-\mu}^{D e-\mu} \frac{d \xi^{c}}{E_{o 1}}+\frac{V_{p}}{2 D e}\left[\Delta^{c} \Delta^{d^{2}} \int_{-\mu}^{D e-\mu} \frac{d \xi^{c}}{\bar{E}_{i n} E_{o 1}}\right. \\
& \left.+\Delta^{c} \int_{-\mu}^{D e-\mu} \frac{\xi^{c^{2}} d \xi^{c}}{\bar{E}_{i n} E_{o 1}}+V^{2} \Delta^{d} \int_{-\mu}^{D e-\mu} \frac{d \xi^{c}}{\bar{E}_{i n} E_{o 1}}\right], \\
\Delta^{c}= & \frac{V_{p} \Delta^{d}}{2 D e} \int_{-\mu}^{D e-\mu} \frac{d \xi^{c}}{E_{o 1}}+\frac{V_{p}}{2 D e}\left(\xi^{d^{2}} \Delta^{d}+\Delta^{d} \Delta^{c 2}+V^{2} \Delta^{c}\right) \\
& \times \int_{-\mu}^{D e-\mu} \frac{d \xi^{c}}{\bar{E}_{i n} E_{o 1}}
\end{aligned}
$$

where one has passed to the thermodynamic limit $(L \longrightarrow$ $\infty)$ and has used the rectangular density of states

$\rho\left(\varepsilon_{\mathbf{k}}^{c}\right)= \begin{cases}\frac{1}{D e}, & \varepsilon_{\mathbf{k}}^{c} \in(0, D e) \\ 0, & \text { otherwise }\end{cases}$
Moreover, one has assumed the d-band to be dispersionless $\left(\xi_{\mathbf{k}}^{d}=\xi^{d}=E^{d}-\mu\right)$. These two equations must be completed with the equation for the average number of electrons in the system per lattice site, namely

$n=-L^{-1} \frac{\partial E_{G}}{\partial \mu}$.

One can deduce that

$$
\begin{aligned}
n^{c}= & 1-\frac{1}{D e} \int_{-\mu}^{D e-\mu} \frac{\xi^{c} d \xi^{c}}{E_{o 1}}-\frac{\xi^{d^{2}}+\Delta^{c 2}}{D e} \int_{-\mu}^{D e-\mu} \frac{\xi^{c} d \xi^{c}}{\bar{E}_{i n} E_{o 1}} \\
& +\frac{V^{2} \xi^{d}}{D e} \int_{-\mu}^{D e-\mu} \frac{d \xi^{c}}{\bar{E}_{i n} E_{o 1}}
\end{aligned}
$$

and

$$
\begin{aligned}
n^{d}= & 1-\frac{\xi^{d}}{D e} \int_{-\mu}^{D e-\mu} \frac{d \xi^{c}}{E_{o 1}}-\frac{\xi^{d}}{D e} \int_{-\mu}^{D e-\mu} \frac{\xi^{c 2} d \xi^{c}}{\bar{E}_{i n} E_{o 1}} \\
& +\frac{V^{2}}{D e} \int_{-\mu}^{D e-\mu} \frac{\xi^{c} d \xi^{c}}{\bar{E}_{i n} E_{o 1}}-\frac{\xi^{d} \Delta^{d^{2}}}{D e} \int_{-\mu}^{D e-\mu} \frac{d \xi^{c}}{\bar{E}_{i n} E_{o 1}} .
\end{aligned}
$$

Of course, $n=n^{c}+n^{d}$ holds.

To solve the problem above, one needs to resort to numerical methods due to the complexity of equations (4.1), (4.2), (4.3), and (4.4). Let us focus on the half-filled case, namely, let $\mu=\frac{D e}{2}$ and $\xi^{d}=0$ then $n^{c}=n^{d}=1$. One has to solve the system of two equations for $\Delta^{c}$ and $\Delta^{d}$. Even this case is not easily tractable. The following set of parameters has been assumed: $D e=0.2 e V$ and $V_{p}=0.1 e V$. As a result, one obtained $\Delta^{c}=0,03609 e V$ and $\Delta^{d}=0.05 e V$ at $V=0$. The critical value of hybridization at which both of gaps vanish amounts to $V_{c}=0.0544 \mathrm{eV}$. Next, we make the comparison of the two-band case with the single-gap one from [25, 26]. In [26], the eigenproblem was solved in the same way as it is done in this paper and the interested reader is referred to one for more details. Now, let us bring the following results from $[25,26]$

$$
\Delta=\sqrt{\frac{D e^{2}}{4 \sinh ^{2}\left(\frac{D e}{U^{c}}\right)}-4 V^{2}}, \quad V_{c}=\frac{1}{4} \frac{D e}{\sinh \left(\frac{D e}{U^{c}}\right)},
$$

where $U^{c}=0.1 \mathrm{eV}$ is the coupling constant of the attractive potential acting between c-electrons. The bandwidth of cband was assumed to be $D e=0.2 e V$. It turns out that $V_{c}=$ $0.0138 e V$ whereas $\Delta(V=0)=0.027572 e V$. Therefore, the critical hybridization in the two-gap case is almost a few times larger than in the single-gap case. The gap at $V=0$ in the single-gap case is visibly lesser than $\Delta^{c}$. Fig. 1 displays $\Delta^{c}, \Delta^{d}$, and $\Delta$ as decreasing functions of hybridization $V$. 
Fig. 1 The dependence of two superconducting gaps on the hybridization $V$ is demonstrated.

For making comparison, the third curve representing the superconducting gap in the single-gap model as a function of the same parameter $V$ has been added. The calculations have been made for

$D e=0.2 e \mathrm{~V}$,

$V_{p}=U^{c}=0.1 e V$

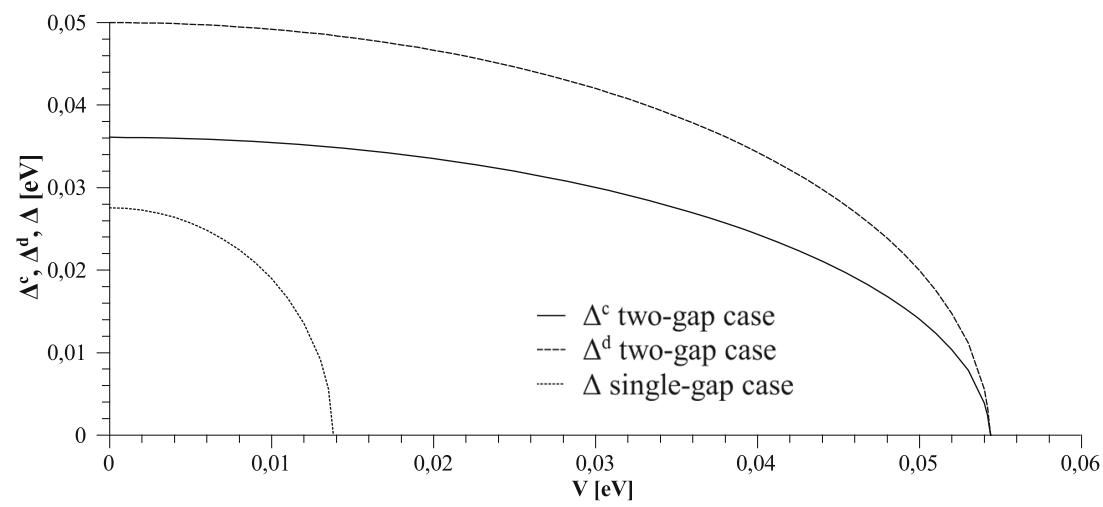

Now, let us determine the free energy per lattice site. By making use of the spectrum, one finds

$\frac{F}{L}=2 \frac{\Delta^{c} \Delta^{d}}{V_{p}}+\frac{1}{L} \sum_{\mathbf{k}}\left(\xi_{\mathbf{k}}^{c}+\xi_{\mathbf{k}}^{d}\right)-\frac{1}{L \beta} \sum_{\mathbf{k}} \ln 2 M_{\mathbf{k}}$,

where

$$
\begin{aligned}
M_{\mathbf{k}}= & 2+2 \cosh \frac{\sqrt{2}}{2} \beta E_{\mathbf{k} m} \\
& +2 \cosh \frac{\sqrt{2}}{2} \beta E_{\mathbf{k} n}+\cosh \beta E_{\mathbf{k} o 1}+\cosh \beta E_{\mathbf{k} o 2}
\end{aligned}
$$

and $\beta=\frac{1}{k T}$. The expression (4.6) will serve us to determine the finite temperature gaps $\Delta^{c}$ and $\Delta^{d}$, namely the conditions given below

$L^{-1} \frac{\partial F}{\partial \Delta^{c}}=0 \quad$ and $\quad L^{-1} \frac{\partial F}{\partial \Delta^{d}}=0$

yield

$$
\begin{gathered}
\frac{2 \Delta^{d}}{V_{p}}=\frac{1}{L} \sum_{\mathbf{k}}\left[\sqrt{2} \frac{\partial E_{\mathbf{k} m}}{\partial \Delta^{c}} F_{\mathbf{k} 1}+\sqrt{2} \frac{\partial E_{\mathbf{k} n}}{\partial \Delta^{c}} F_{\mathbf{k} 2}\right. \\
\left.+\frac{\partial E_{\mathbf{k} o 1}}{\partial \Delta^{c}} F_{\mathbf{k} 3}+\frac{\partial E_{\mathbf{k} o 2}}{\partial \Delta^{c}} F_{\mathbf{k} 4}\right], \\
\frac{2 \Delta^{c}}{V_{p}}=\frac{1}{L} \sum_{\mathbf{k}}\left[\sqrt{2} \frac{\partial E_{\mathbf{k} m}}{\partial \Delta^{d}} F_{\mathbf{k} 1}+\sqrt{2} \frac{\partial E_{\mathbf{k} n}}{\partial \Delta^{d}} F_{\mathbf{k} 2}\right. \\
\left.+\frac{\partial E_{\mathbf{k} o 1}}{\partial \Delta^{d}} F_{\mathbf{k} 3}+\frac{\partial E_{\mathbf{k} o 2}}{\partial \Delta^{d}} F_{\mathbf{k} 4}\right],
\end{gathered}
$$

where

$$
\frac{\partial E_{\mathbf{k} o 1 / 2}}{\partial \Delta^{c}}=\frac{\Delta^{c}}{E_{\mathbf{k} o 1 / 2}} \pm \frac{\Delta^{c} \xi_{\mathbf{k}}^{c 2}+\Delta^{c} \Delta^{d^{2}}+\Delta^{d} V^{2}}{\bar{E}_{i n} E_{\mathbf{k} o 1 / 2}}
$$

$$
\begin{aligned}
& \frac{\partial E_{\mathbf{k} n / m}}{\partial \Delta^{c}}=\frac{\Delta^{c}}{E_{\mathbf{k} n / m}} \\
& \pm \frac{-\Delta^{c} \xi_{\mathbf{k}}^{c 2}+\Delta^{c} \xi_{\mathbf{k}}^{d^{2}}-\Delta^{c} \Delta^{d^{2}}-2 \Delta^{d} V^{2}+2 \Delta^{c} V^{2}+\Delta^{c 3}}{E_{i n} E_{\mathbf{k} n / m}}
\end{aligned}
$$

$$
\begin{aligned}
& \frac{\partial E_{\mathbf{k} o 1 / 2}}{\partial \Delta^{d}}=\frac{\Delta^{d}}{E_{\mathbf{k} o 1 / 2}} \pm \frac{\Delta^{d} \xi_{\mathbf{k}}^{d^{2}}+\Delta^{d} \Delta^{c 2}+\Delta^{c} V^{2}}{\bar{E}_{i n} E_{\mathbf{k} o 1 / 2}} \\
& \frac{\partial E_{\mathbf{k} n / m}}{\partial \Delta^{d}}=\frac{\Delta^{d}}{E_{\mathbf{k} n / m}} \\
& \pm \frac{\Delta^{d} \xi_{\mathbf{k}}^{c^{2}}-\Delta^{d} \xi_{\mathbf{k}}^{d^{2}}-\Delta^{d} \Delta^{c 2}+2 \Delta^{d} V^{2}-2 \Delta^{c} V^{2}+\Delta^{d^{3}}}{E_{i n} E_{\mathbf{k} n / m}}
\end{aligned}
$$

and the functions

$$
\begin{aligned}
& F_{\mathbf{k} 1}=\frac{\sinh \frac{\sqrt{2}}{2} \beta E_{\mathbf{k} m}}{M_{\mathbf{k}}}, \quad F_{\mathbf{k} 2}=\frac{\sinh \frac{\sqrt{2}}{2} \beta E_{\mathbf{k} n}}{M_{\mathbf{k}}}, \\
& F_{\mathbf{k} 3}=\frac{\sinh \beta E_{\mathbf{k} o 1}}{M_{\mathbf{k}}}, \quad F_{\mathbf{k} 4}=\frac{\sinh \beta E_{\mathbf{k} o 2}}{M_{\mathbf{k}}} .
\end{aligned}
$$

The average number of electrons per lattice site can be obtained owing to the following condition $n=-\frac{1}{L} \frac{\partial F}{\partial \mu}$ that yields

$$
\begin{aligned}
n= & \frac{1}{L} \sum_{\mathbf{k}} 2+\frac{1}{L} \sum_{\mathbf{k}}\left[\sqrt{2} \frac{\partial E_{\mathbf{k} m}}{\partial \mu} F_{\mathbf{k} 1}+\sqrt{2} \frac{\partial E_{\mathbf{k} n}}{\partial \mu} F_{\mathbf{k} 2}\right. \\
& \left.+\frac{\partial E_{\mathbf{k} o 1}}{\partial \mu} F_{\mathbf{k} 3}+\frac{\partial E_{\mathbf{k} o 2}}{\partial \mu} F_{\mathbf{k} 4}\right]
\end{aligned}
$$

with the derivatives

$$
\begin{aligned}
& \frac{\partial E_{\mathbf{k} o 1 / 2}}{\partial \mu}=-\frac{\xi_{\mathbf{k}}^{c}+\xi_{\mathbf{k}}^{d}}{E_{\mathbf{k} o 1 / 2}} \\
& \pm \frac{-\xi_{\mathbf{k}}^{c} \xi_{\mathbf{k}}^{d^{2}}-\xi_{\mathbf{k}}^{d} \xi_{\mathbf{k}}^{c 2}-\xi_{\mathbf{k}}^{c} \Delta^{c 2}-\xi_{\mathbf{k}}^{d} \Delta^{d^{2}}+V^{2}\left(\xi_{\mathbf{k}}^{c}+\xi_{\mathbf{k}}^{d}\right)}{\bar{E}_{i n} E_{\mathbf{k} o 1 / 2}}
\end{aligned}
$$

and

$$
\frac{\partial E_{\mathbf{k} n / m}}{\partial \mu}=-\frac{\xi_{\mathbf{k}}^{c}+\xi_{\mathbf{k}}^{d}}{E_{\mathbf{k} n / m}} \pm \frac{-\xi_{\mathbf{k}}^{c 3}-\xi_{\mathbf{k}}^{d^{3}}+\xi_{\mathbf{k}}^{c} \xi_{\mathbf{k}}^{d^{2}}+\xi_{\mathbf{k}}^{d} \xi_{\mathbf{k}}^{c 2}+\frac{1}{2}\left(\xi_{\mathbf{k}}^{c}-\xi_{\mathbf{k}}^{d}\right)\left(\Delta^{c 2}-\Delta^{d^{2}}\right)-4 V^{2}\left(\xi_{\mathbf{k}}^{c}+\xi_{\mathbf{k}}^{d}\right)}{E_{i n} E_{\mathbf{k} n / m}}
$$


Fig. 2 The dependence of the critical temperature on the hybridization $V$ is demonstrated. Two curves are presented-one for the two-gap model and the other for the single-gap one. The calculations have been made for $D e=0.2 \mathrm{eV}$, $V_{p}=U^{c}=0.1 e V$

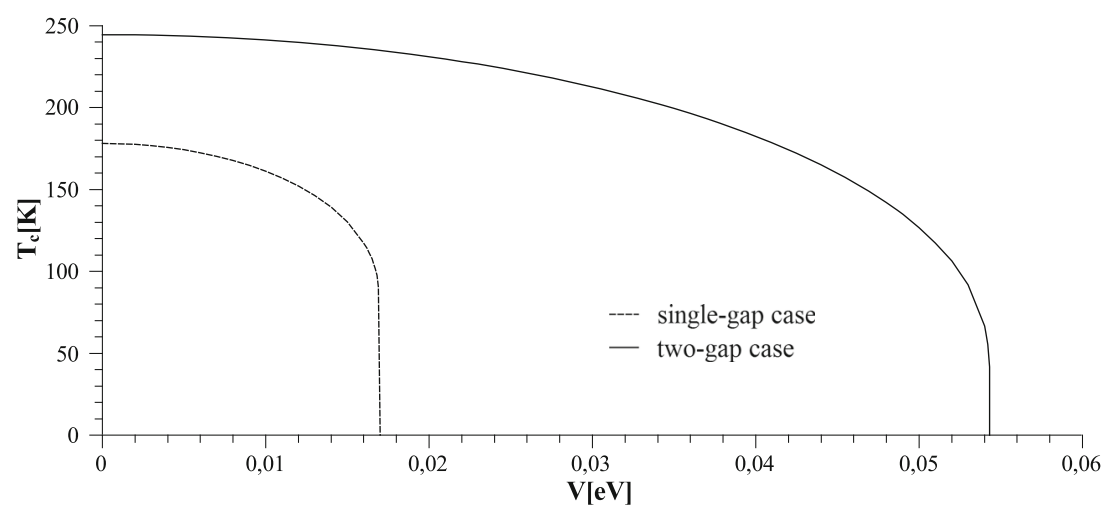

The sign "+ " corresponds to the indices " 1 " and " $n$ " while " - " to " 2 " and " $m$ ".

The pair of equations (4.7) and (4.8) will serve us to find the equation for the critical temperature $T_{c}$ that for $\Delta^{c}=0$, $\Delta^{d}=0, \xi_{\mathbf{k}}^{d}=0$, and $\mu=\frac{D e}{2}$ ( the half-filled case) reads

$1=\frac{I^{2}}{I^{c} I^{d}}$,

where

$$
\begin{gathered}
I=\frac{2}{V_{p}}+\frac{1}{D e} \int_{-\frac{D e}{2}}^{\frac{D e}{2}} d \xi^{c}\left[\frac{2 \sqrt{2} V^{2}}{E_{i n} E_{m}} F_{1}-\frac{2 \sqrt{2} V^{2}}{E_{i n} E_{n}}\right. \\
\left.\times F_{2}-\frac{V^{2}}{\bar{E}_{i n} E_{o 1}} F_{3}-\frac{V^{2}}{\bar{E}_{i n} E_{o 2}} F_{4}\right], \\
I^{c}=\frac{1}{D e} \int_{-\frac{D e}{2}}^{\frac{D e}{2}} d \xi^{c}\left[\sqrt{2}\left(\frac{1}{E_{m}}+\frac{2 V^{2}-\xi^{c 2}}{E_{i n} E_{m}}\right)\right. \\
\left.+\left(\frac{1}{E_{o 1}}+\frac{\xi^{c 2}}{\bar{E}_{i n} E_{o 1}}\right) F_{3}+\left(\frac{1}{E_{o 2}}-\frac{\xi^{c 2}}{\bar{E}_{i n} E_{o 2}}\right) F_{4}\right], \\
I^{d}=\frac{1}{D e} \int_{-\frac{D e}{2}}^{\frac{D e}{2}} d \xi^{c}\left[\sqrt{2}\left(\frac{1}{E_{n}}+\frac{2 V^{2}+\xi^{c 2}}{E_{i n} E_{m}}\right) F_{1}\right. \\
\left.+\sqrt{2}\left(\frac{1}{E_{n}}-\frac{2 V^{2}+\xi^{c 2}}{E_{i n} E_{n}}\right) F_{2}+\frac{F_{3}}{E_{o 1}}+\frac{F_{4}}{E_{o 2}}\right],
\end{gathered}
$$

with

$$
\begin{aligned}
E_{o 1} & =\sqrt{\xi^{c^{2}}+4 V^{2}}, \quad E_{o 2}=\left|\xi^{c}\right|, \quad \bar{E}_{i n}=V^{2} \\
E_{m} & =\frac{\sqrt{2}}{2}\left(\sqrt{\xi^{c^{2}}+2 V^{2}}-\left|\xi^{c}\right|\right), \\
E_{n} & =\frac{\sqrt{2}}{2}\left(\left|\xi^{c}\right|+\sqrt{\xi^{c^{2}}+2 V^{2}}\right), \\
E_{i n} & =\left|\xi^{c}\right| \sqrt{\xi^{c^{2}}+4 V^{2}} .
\end{aligned}
$$

The graph of the critical temperature in the two-gap case versus the single-particle hybridization $V$ is shown on Fig. 2. The calculations were done for the same set of parameters as in the case of the ground state. It is seen that $T_{c}$ is a decreasing function of $V$. Initially, for small values of $V$, the decrease is not rapid but in the vicinity of $V=0.05432 \mathrm{eV}$ at which $T_{c}=0$ this becomes very fast. Note that for $V=0 T_{c}=244.61 \mathrm{~K}$, and this value can be numerically found from the following equation

$$
\frac{4 D e}{V_{p}^{2} \beta_{c}}=\int_{0}^{\frac{D e}{2}} \frac{d \xi^{c}}{\xi^{c}} \tanh \frac{1}{2} \beta_{c} \xi^{c}
$$

that can be easily derived. The single-particle hybridization is a strong factor reducing this temperature. One can see from Fig. 2 the effectiveness of the competition of singleparticle hybridization with the pair exchange mechanism of superconductivity. The influence of the single-particle hybridization on the critical temperature in the case of the single-gap case was investigated as well. The comparison of both of cases is shown on Fig. 2. The curve representing this case is similar in shape to that for the two-gap one but $T_{c}=178.19 \mathrm{~K}$ for $V=0$ as well as $V=0.017 \mathrm{eV}$ at which $T_{c}=0$ are lesser than in the former one. The equation for $T_{c}$ reads

$\frac{2 D e}{U^{c}}=\int_{-\frac{D e}{2}}^{\frac{D e}{2}} d \xi^{c}\left[\left(\frac{1}{E_{V}}-\frac{1}{\left|\xi^{c}\right|}\right) F_{1}+\left(\frac{1}{E_{V}}+\frac{1}{\left|\xi^{c}\right|}\right) F_{2}+\frac{F_{3}}{\left|\xi^{c}\right|}+\frac{F_{4}}{E_{V}}\right]$ 
where $F_{i}$ for $i=1, . ., 4$ are the same as the functions (4.9) with $E_{V}=E_{o 1}, E_{o 2}=\left|\xi^{c}\right|$ and $E_{m}$ in $F_{1}$ along with $E_{n}$ in $F_{2}$ identical to (4.13) while $U^{c}=0.1 \mathrm{eV}$.

\section{Conclusions}

The aim of this paper was to get an effective two-band polaronic Hamiltonian combining superconductivity and features of the Kondo lattice model via the removal of local phonon-electron interactions by making use of two canonical transformations. In this way, one has obtained a system in which beside effective intraband and interband Coulomb potentials a pair-exchange interaction and an anisotropic Kondo-lattice component have appeared. Due to this at least superconductivity and Kondo effect compete with one another and it is not excluded that other kinds of ordering are possible. It is important that the single-particle hybridization is still present in the final Hamiltonian. A density-dependent hybridization term is quite new one. Moreover, a new hopping term describing the intersite transfer of polarons from different bands appeared as well. All these aforementioned terms make the new Hamiltonian comprise features of a few models. These are the anisotropic Kondo-latticemodel, the periodic Anderson model, the d-p model, and the so-called two-component model with local pairs $[3,5]$.

The results point to much greater importance of the phonon-electron interactions in new materials such as for instance cuprates and heavy fermions. They can lead to the Kondo effect or magnetism on its own provided that beside the intraband phonon-electron potentials the interband one is taken into account as well.

The Hamiltonian (2.8) describes an extremely complex many-particle system. The investigation of the general form poses a very challenging task but can bring in new insight into the understanding of the interplay between superconductivity and other phenomena in some substances. That is why in this paper only one simplified situation has been considered. The simplification consisted in rejecting all effective interactions apart from the so-called pair-exchange potential and the single-electron hybridization. It has been shown that there are two gaps in this system and they vanish if the hybridization increases. Additionally, it has been demonstrated that the critical temperature decreases as well. The next step will be the incorporation of neglected interactions into considerations. Now, the case with $\xi^{d}$ of a finite width and the interband hopping is being investigated. The spin-spin interactions also seem especially interesting for this purpose.

Open Access This article is distributed under the terms of the Creative Commons Attribution License which permits any use, distribution, and reproduction in any medium, provided the original author(s) and the source are credited.

\section{Appendix}

The transformed Hamiltonian $\bar{H}_{F}$ is obtained via the application of the transformation $U_{2}$. It reads

$$
\begin{aligned}
& \bar{H}_{F}=\sum_{i, j \sigma}\left[t_{i j}^{c} A_{i j}^{++}-t_{i j}^{d} A_{i j}^{--}\right] c_{i \sigma}^{*} c_{j \sigma} \\
& +\sum_{i, j \sigma}\left[t_{i j}^{d} A_{i j}^{++}-t_{i j}^{c} A_{i j}^{--}\right] d_{i \sigma}^{*} d_{j \sigma}+\sum_{i, j \sigma}\left[t_{i j}^{d} A_{i j}^{-+}\right. \\
& \left.-t_{i j}^{c} A_{i j}^{+-}\right] c_{i \sigma}^{*} d_{j \sigma}+ \\
& +\sum_{i, j \sigma}\left[t_{i j}^{c} A_{i j}^{-+}-t_{i j}^{d} A_{i j}^{+-}\right] d_{i \sigma}^{*} c_{j \sigma}+\sum_{i \sigma} \bar{E}_{i}^{c} n_{i \sigma}^{c}+\sum_{i \sigma} \bar{E}_{i}^{d} n_{i \sigma}^{d} \\
& +\left(E^{d}-E^{c}\right) \sum_{i \sigma} A_{i}^{3}\left(c_{i \sigma}^{*} d_{i \sigma}-d_{i \sigma}^{*} c_{i \sigma}\right)+ \\
& +\sum_{i} \bar{U}_{i}^{c} n_{i+}^{c} n_{i-}^{c}+\sum_{i} \bar{U}_{i}^{d} n_{i+}^{d} n_{i-}^{d} \\
& +\sum_{i} U_{i 1}^{c d}\left(n_{i+}^{c} n_{i-}^{d}+n_{i+}^{d} n_{i-}^{c}\right)+\sum_{i \sigma} U_{i 2}^{c d} n_{i \sigma}^{c} n_{i \sigma}^{d}+ \\
& +\tilde{U}^{c} \sum_{i \sigma}\left(A_{i}^{7} n_{i \sigma}^{c}-A_{i}^{8} n_{i \sigma}^{d}\right)\left(d_{i-\sigma}^{*} c_{i-\sigma}-c_{i-\sigma}^{*} d_{i-\sigma}\right) \\
& +\tilde{U}^{d} \sum_{i \sigma}\left(A_{i}^{8} n_{i \sigma}^{c}-A_{i}^{7} n_{i \sigma}^{d}\right)\left(d_{i-\sigma}^{*} c_{i-\sigma}-c_{i-\sigma}^{*} d_{i-\sigma}\right)+
\end{aligned}
$$$$
\begin{aligned}
+\bar{V} \sum_{i \sigma}\left(d_{i \sigma}^{*} c_{i \sigma}+c_{i \sigma}^{*} d_{i \sigma}\right)- & 2 \frac{g g^{c d}}{\omega} \sum_{i \sigma}\left(n_{i \sigma}^{c}+n_{i \sigma}^{d}\right) \\
& \times\left(d_{i \sigma}^{*} c_{i \sigma}+c_{i \sigma}^{*} d_{i \sigma}\right)+
\end{aligned}
$$$$
+\tilde{U}^{c d} \sum_{i \sigma}\left(A_{i}^{8}+A_{i}^{7}\right)\left(n_{i \sigma}^{d}-n_{i \sigma}^{c}\right)\left(d_{i \sigma}^{*} c_{i \sigma}-c_{i \sigma}^{*} d_{i \sigma}\right)
$$$$
+\sum_{i} \bar{V}_{p i}\left(c_{i+}^{*} c_{i-}^{*} d_{i-} d_{i+}+d_{i+}^{*} d_{i-}^{*} c_{i-} c_{i+}\right)+
$$$$
+\sum_{i} \bar{J}_{i}^{ \pm}\left(c_{i+}^{*} c_{i-} d_{i-}^{*} d_{i+}+d_{i+}^{*} d_{i-} c_{i-}^{*} c_{i+}\right)+\omega \sum_{i} b_{i}^{*} b_{i} .
$$

Some terms are modified by exponential factors involving the lattice deformation. These factors appear owing to the mutual multiplications of new operators in the resulting Hamiltonian: $\bar{c}_{i \sigma}^{*}, \bar{c}_{i \sigma}, \bar{d}_{i \sigma}^{*}$, and $\bar{d}_{i \sigma}$. The operators are combinations of terms containing $\cosh \left[\frac{g^{c d}}{\omega}\left(b_{i}^{*}-b_{i}\right)\right]$ and 
$\sinh \left[\frac{g^{c d}}{\omega}\left(b_{i}^{*}-b_{i}\right)\right]$. Let us start with those for hoppings, namely,

$$
\begin{aligned}
& A_{i j}^{++}=e^{\frac{g}{\omega}\left(b_{i}^{*}-b_{i}\right)} e^{-\frac{g}{\omega}\left(b_{j}^{*}-b_{j}\right)} \cosh \left[\frac{g^{c d}}{\omega}\left(b_{i}^{*}-b_{i}\right)\right] \\
& \times \cosh \left[\frac{g^{c d}}{\omega}\left(b_{j}^{*}-b_{j}\right)\right]= \\
& =\frac{1}{4}\left[e^{\frac{g^{c d}+g}{\omega}\left(b_{i}^{*}-b_{i}\right)}+e^{\frac{g-g^{c d}}{\omega}\left(b_{i}^{*}-b_{i}\right)}\right] \\
& \times\left[e^{-\frac{g-g}{\omega}\left(b_{j}^{*}-b_{j}\right)}+e^{-\frac{g^{c d}+g}{\omega}\left(b_{j}^{*}-b_{j}\right)}\right], \\
& A_{i j}^{--}=e^{\frac{g}{\omega}\left(b_{i}^{*}-b_{i}\right)} e^{-\frac{g}{\omega}\left(b_{j}^{*}-b_{j}\right)} \sinh \left[\frac{g^{c d}}{\omega}\left(b_{i}^{*}-b_{i}\right)\right] \\
& \times \sinh \left[\frac{g^{c d}}{\omega}\left(b_{j}^{*}-b_{j}\right)\right]= \\
& =\frac{1}{4}\left[e^{\frac{g^{c d}+g}{\omega}\left(b_{i}^{*}-b_{i}\right)}-e^{\frac{g-g^{c d}}{\omega}\left(b_{i}^{*}-b_{i}\right)}\right] \\
& \times\left[e^{-\frac{g-g c d}{\omega}\left(b_{j}^{*}-b_{j}\right)}-e^{-\frac{g^{c d}+g}{\omega}\left(b_{j}^{*}-b_{j}\right)}\right], \\
& A_{i j}^{+-}=e^{\frac{g}{\omega}\left(b_{i}^{*}-b_{i}\right)} e^{-\frac{g}{\omega}\left(b_{j}^{*}-b_{j}\right)} \times \cosh \left[\frac{g^{c d}}{\omega}\left(b_{i}^{*}-b_{i}\right)\right] \\
& \times \sinh \left[\frac{g^{c d}}{\omega}\left(b_{j}^{*}-b_{j}\right)\right]= \\
& =\frac{1}{4}\left[e^{\frac{g^{c d}+g}{\omega}\left(b_{i}^{*}-b_{i}\right)}+e^{\frac{g-g c d}{\omega}\left(b_{i}^{*}-b_{i}\right)}\right] \\
& \times\left[e^{-\frac{g-g^{c d}}{\omega}\left(b_{j}^{*}-b_{j}\right)}-e^{-\frac{g^{c d}+g}{\omega}\left(b_{j}^{*}-b_{j}\right)}\right], \\
& A_{i j}^{-+}=e^{\frac{g}{\omega}\left(b_{i}^{*}-b_{i}\right)} e^{-\frac{g}{\omega}\left(b_{j}^{*}-b_{j}\right)} \sinh \left[\frac{g^{c d}}{\omega}\left(b_{i}^{*}-b_{i}\right)\right] \\
& \times \cosh \left[\frac{g^{c d}}{\omega}\left(b_{j}^{*}-b_{j}\right)\right]= \\
& =\frac{1}{4}\left[e^{\frac{g^{c d}+g}{\omega}\left(b_{i}^{*}-b_{i}\right)}-e^{\frac{g-g}{\omega}\left(b_{i}^{*}-b_{i}\right)}\right] \\
& \times\left[e^{-\frac{g-g^{c d}}{\omega}\left(b_{j}^{*}-b_{j}\right)}+e^{-\frac{g^{c d}+g}{\omega}\left(b_{j}^{*}-b_{j}\right)}\right] .
\end{aligned}
$$

The remaining factors are as follows

$$
\begin{aligned}
& A_{i}^{1}=\cosh \left[\frac{g^{c d}}{\omega}\left(b_{i}^{*}-b_{i}\right)\right]^{2} \\
& =\frac{1}{4}\left(e^{\frac{2 g^{c d}}{\omega}\left(b_{i}^{*}-b_{i}\right)}+2+e^{\frac{-2 g^{c d}}{\omega}\left(b_{i}^{*}-b_{i}\right)}\right),
\end{aligned}
$$

$$
\begin{aligned}
& A_{i}^{2}=\times \sinh \left[\frac{g^{c d}}{\omega}\left(b_{i}^{*}-b_{i}\right)\right]^{2} \\
& =\frac{1}{4}\left(e^{\frac{2 g^{c d}}{\omega}\left(b_{i}^{*}-b_{i}\right)}-2+e^{\frac{-2 g^{c d}}{\omega}\left(b_{i}^{*}-b_{i}\right)}\right), \\
& A_{i}^{3}=\cosh \left[\frac{g^{c d}}{\omega}\left(b_{i}^{*}-b_{i}\right)\right] \sinh \left[\frac{g^{c d}}{\omega}\left(b_{i}^{*}-b_{i}\right)\right] \\
& =\frac{1}{4}\left(e^{\frac{2 g c d}{\omega}\left(b_{i}^{*}-b_{i}\right)}-e^{\frac{-2 g c d}{\omega}\left(b_{i}^{*}-b_{i}\right)}\right), \\
& A_{i}^{4}=\cosh \times\left[\frac{g^{c d}}{\omega}\left(b_{i}^{*}-b_{i}\right)\right]^{4}=\frac{1}{16}\left(e^{\frac{{ }^{\frac{g}{c d}}}{\omega}\left(b_{i}^{*}-b_{i}\right)}\right. \\
& \left.+6+e^{\frac{-4 g^{c d}}{\omega}\left(b_{i}^{*}-b_{i}\right)}+4 e^{\frac{2 g c d}{\omega}\left(b_{i}^{*}-b_{i}\right)}+4 e^{\frac{-2 g^{c d}}{\omega}\left(b_{i}^{*}-b_{i}\right)}\right), \\
& A_{i}^{5}=\sinh \times\left[\frac{g^{c d}}{\omega}\left(b_{i}^{*}-b_{i}\right)\right]^{4} \\
& =\frac{1}{16}\left(e^{\frac{4 g^{c d}}{\omega}\left(b_{i}^{*}-b_{i}\right)}+6+e^{\frac{-4 g^{c d}}{\omega}\left(b_{i}^{*}-b_{i}\right)}-4 e^{\frac{2 g^{c d}}{\omega}\left(b_{i}^{*}-b_{i}\right)}\right. \\
& \left.-4 e^{\frac{-2 g^{c d}}{\omega}\left(b_{i}^{*}-b_{i}\right)}\right), \\
& A_{i}^{6}=\cosh \left[\frac{g^{c d}}{\omega}\left(b_{i}^{*}-b_{i}\right)\right]^{2} \sinh \left[\frac{g^{c d}}{\omega}\left(b_{i}^{*}-b_{i}\right)\right]^{2} \\
& =\frac{1}{16}\left(e^{\frac{4 g^{c d}}{\omega}\left(b_{i}^{*}-b_{i}\right)}-2+e^{\frac{-4 g^{c d}}{\omega}\left(b_{i}^{*}-b_{i}\right)}\right), \\
& A_{i}^{7}=\cosh \left[\frac{g^{c d}}{\omega}\left(b_{i}^{*}-b_{i}\right)\right]^{3} \sinh \left[\frac{g^{c d}}{\omega}\left(b_{i}^{*}-b_{i}\right)\right]= \\
& =\frac{1}{16}\left(e^{\frac{4 g^{c d}}{\omega}\left(b_{i}^{*}-b_{i}\right)}-e^{\frac{-4 g c d}{\omega}\left(b_{i}^{*}-b_{i}\right)}\right. \\
& \left.+2 e^{\frac{2 g^{c d}}{\omega}\left(b_{i}^{*}-b_{i}\right)}-2 e^{\frac{-2 g^{c d}}{\omega}\left(b_{i}^{*}-b_{i}\right)}\right), \\
& A_{i}^{8}=\cosh \left[\frac{g^{c d}}{\omega}\left(b_{i}^{*}-b_{i}\right)\right] \sinh \left[\frac{g^{c d}}{\omega}\left(b_{i}^{*}-b_{i}\right)\right]^{3}= \\
& =\frac{1}{16}\left(e^{\frac{4 g c d}{\omega}\left(b_{i}^{*}-b_{i}\right)}-e^{\frac{-4 g c d}{\omega}\left(b_{i}^{*}-b_{i}\right)}-2 e^{\frac{2 g^{c d}}{\omega}\left(b_{i}^{*}-b_{i}\right)}\right. \\
& \left.+2 e^{\frac{-2 g^{c d}}{\omega}\left(b_{i}^{*}-b_{i}\right)}\right) .
\end{aligned}
$$

Note that $A_{i}^{1^{2}}=A_{i}^{4}, A_{i}^{1} A_{i}^{2}=A_{i}^{6}, A_{i}^{1} A_{i}^{3}=A_{i}^{7}, A_{i}^{3} A_{i}^{2}=$ $A_{i}^{8}, A_{i}^{2^{2}}=A_{i}^{5}$, and $A_{i}^{3^{2}}=A_{i}^{6}$. The other factors are as follows

$$
\begin{gathered}
\bar{E}_{i}^{c}=\tilde{E}^{c} A_{i}^{1}-\tilde{E}^{d} A_{i}^{2}-\tilde{U}^{c d} A_{i}^{2}-\frac{g^{c d^{2}}}{\omega}, \\
\bar{E}_{i}^{d}=\tilde{E}^{d} A_{i}^{1}-\tilde{E}^{c} A_{i}^{2}-\tilde{U}^{c d} A_{i}^{2}-\frac{g^{c d^{2}}}{\omega}, \\
\bar{U}_{i}^{c}=\tilde{U}^{c} A_{i}^{4}+\tilde{U}^{d} A_{i}^{5}-2 \tilde{U}^{c d} A_{i}^{6}, \\
\bar{U}_{i}^{d}=\tilde{U}^{c} A_{i}^{5}+\tilde{U}^{d} A_{i}^{4}-2 \tilde{U}^{c d} A_{i}^{6},
\end{gathered}
$$




$$
\begin{aligned}
\bar{U}_{i 1}^{c d} & =\tilde{U}^{c d}\left(A_{i}^{4}+A_{i}^{5}\right)-\left(\tilde{U}^{c}+\tilde{U}^{d}\right) A_{i}^{6}, \\
\bar{U}_{i 2}^{c d} & =\tilde{U}^{c d}\left(A_{i}^{1}+A_{i}^{2}\right)+2 \frac{g^{c d^{2}}}{\omega}, \\
\bar{V}_{p i} & =\left(\tilde{U}^{c}+\tilde{U}^{c}-2 \tilde{U}^{c d}\right) A_{i}^{6}-2 \frac{g^{c d^{2}}}{\omega}, \\
\bar{J}_{i}^{ \pm} & =\left(\tilde{U}^{c}+\tilde{U}^{c}-2 \tilde{U}^{c d}\right) A_{i}^{6}+2 \frac{g^{c d^{2}}}{\omega} .
\end{aligned}
$$

Now having found these factors one could determine the averages of them over the grand canonical ensemble of free phonons

$\rho=\frac{e^{-\beta H_{p h}}}{\operatorname{Tr} e^{-\beta H_{p h}}}$,

where $H_{p h}=\omega \sum_{i} b_{i}^{*} b_{i}$ end $\beta=\frac{1}{k T}$. On knowing that at low temperatures for an arbitrary $\alpha \operatorname{Tr}\left(\rho e^{ \pm \alpha\left(b_{i}^{*}-b_{i}\right)}\right)=$ $e^{-\frac{1}{2} \alpha^{2} \operatorname{coth} \frac{\beta \omega}{2}} \approx e^{-\frac{1}{2} \alpha^{2}}$ holds, one finds for the arbitrary $g^{c d}$

$$
\begin{aligned}
& \operatorname{Tr} \rho A_{i j}^{++}=\frac{1}{4}\left[e^{-\frac{\left(g-g^{c d}\right)^{2}}{2 \omega^{2}}}+e^{-\frac{\left(g^{c d}+g\right)^{2}}{2 \omega^{2}}}\right]^{2}, \\
& \operatorname{Tr} \rho A_{i j}^{--}=\frac{1}{4}\left[e^{-\frac{(g-g c d}{2 \omega^{2}}}-e^{\left.-\frac{(g c d}{2 \omega^{2}}\right)^{2}}\right]^{2}, \\
& \operatorname{Tr} \rho A_{i j}^{+-}=\frac{1}{4}\left(e^{-\frac{(g-g c d}{\omega^{2}}}-e^{-\frac{\left(g^{c d}+g\right)^{2}}{\omega^{2}}}\right), \\
& \operatorname{Tr} \rho A_{i j}^{-+}=\frac{1}{4}\left(e^{-\frac{(g+g c d}{\omega^{2}}}-e^{-\frac{(g-g c d}{\omega^{2}}}\right) \\
& \operatorname{Tr} \rho A_{i}^{1}=\frac{1}{2}+\frac{1}{2} e^{\frac{-2 g}{\omega^{2}}}, \quad \operatorname{Tr} \rho A_{i}^{2}=-\frac{1}{2}+\frac{1}{2} e^{\frac{-2 g c^{2}}{\omega^{2}}}, \\
& \operatorname{Tr} \rho A_{i}^{3}=0
\end{aligned}
$$$$
\operatorname{Tr} \rho A_{i}^{4}=\frac{1}{8}\left(e^{-\frac{8 g c d^{2}}{\omega^{2}}}+3+4 e^{-\frac{2 g c d^{2}}{\omega^{2}}}\right),
$$$$
\operatorname{Tr} \rho A_{i}^{5}=\frac{1}{8}\left(e^{-\frac{8 g c d^{2}}{\omega^{2}}}+3-4 e^{-\frac{2 g c d^{2}}{\omega^{2}}}\right),
$$$$
\operatorname{Tr} \rho A_{i}^{6}=2 e^{-\frac{8 g \text { g } d^{2}}{\omega^{2}}}-2, \quad \operatorname{Tr} \rho A_{i}^{7}=\operatorname{Tr} \rho A_{i}^{8}=0 .
$$

Since we are working in the strong coupling regime, it is $\frac{g}{\omega} \gg 1$ and $\frac{g^{c d}}{\omega} \gg 1$ then we can neglect terms such as $e^{-\frac{(g c d+g)^{2}}{2 \omega^{2}}}, e^{-\frac{8 g c d^{2}}{\omega^{2}}}$, and $e^{-\frac{2 g c d^{2}}{\omega^{2}}}$. Thus, in this limit, one obtains

$$
\begin{array}{r}
\operatorname{Tr} \rho A_{i j}^{++}=\operatorname{Tr} \rho A_{i j}^{--}=\operatorname{Tr} \rho A_{i j}^{+-}=\frac{1}{4} e^{-\frac{\left(g-g^{c d}\right)^{2}}{2 \omega^{2}}}, \\
\operatorname{Tr} \rho A_{i j}^{-+}=-\operatorname{Tr} \rho A_{i j}^{+-},
\end{array}
$$

$\operatorname{Tr} \rho A_{i}^{1}=\frac{1}{2}, \quad \operatorname{Tr} \rho A_{i}^{2}=-\frac{1}{2}$,

$\operatorname{Tr} \rho A_{i}^{3}=\operatorname{Tr} \rho A_{i}^{7}=\operatorname{Tr} \rho A_{i}^{8}=0$,
$\operatorname{Tr} \rho A_{i}^{4}=\operatorname{Tr} \rho A_{i}^{5}=\frac{3}{8}, \quad \operatorname{Tr} \rho A_{i}^{6}=-\frac{1}{8}$.

This approximation has been applied to obtain the Hamiltonian (2.8). It is worthwhile to add that the Ising term with $S_{i}^{z c} S_{i}^{z d}$ was incorporated by making use of the expression $n_{i+}^{c} n_{i+}^{d}+n_{i-}^{c} n_{i-}^{d}=4 S_{i}^{z c} S_{i}^{z d}+$ $n_{i+}^{c} n_{i-}^{d}+n_{i-}^{c} n_{i+}^{d}$. In opposite limit $g^{c d} \rightarrow 0$, one gets

$$
\begin{aligned}
\operatorname{Tr} \rho A_{i j}^{++} & =e^{-\left(\frac{g}{\omega}\right)^{2}}, \quad \operatorname{Tr} \rho A_{i j}^{--}=\operatorname{Tr} \rho A_{i j}^{+-}=\operatorname{Tr} \rho A_{i j}^{-+}=0, \\
\operatorname{Tr} \rho A_{i}^{1} & =\operatorname{Tr} \rho A_{i}^{4}=1, \operatorname{Tr} \rho A_{i}^{2}=\operatorname{Tr} \rho A_{i}^{3}=\operatorname{Tr} \rho A_{i}^{5}=\operatorname{Tr} \rho A_{i}^{6} \\
& =\operatorname{Tr} \rho A_{i}^{7}=\operatorname{Tr} \rho A_{i}^{8}=0 .
\end{aligned}
$$

Thus, after averaging the Hamiltonian $\bar{H}_{F}$ over the grand canonical ensamble of free phonons one obtains:

$t_{i j}^{c} \operatorname{Tr} \rho A_{i j}^{++}-t_{i j}^{d} \operatorname{Tr} \rho A_{i j}^{--}=t_{i j}^{c}, \quad t_{i j}^{d} \operatorname{Tr} \rho A_{i j}^{++}-t_{i j}^{c} \operatorname{Tr} \rho A_{i j}^{--}=t_{i j}^{d}$, $t_{i j}^{d} \operatorname{Tr} \rho A_{i j}^{-+}-t_{i j}^{c} \operatorname{Tr} \rho A_{i j}^{+-}=t_{i j}^{c} \operatorname{Tr} \rho A_{i j}^{-+}-t_{i j}^{d} \operatorname{Tr} \rho A_{i j}^{+-}=0$, $\operatorname{Tr} \rho \bar{E}_{i}^{c}=\tilde{E}^{c}, \quad \operatorname{Tr} \rho \bar{E}_{i}^{d}=\tilde{E}^{d}, \quad \operatorname{Tr} \rho \bar{U}_{i}^{c}=\tilde{U}^{c}, \quad \operatorname{Tr} \rho \bar{U}_{i}^{d}=\tilde{U}^{d}$, $\operatorname{Tr} \rho \bar{U}_{i 1}^{c d}=\operatorname{Tr} \rho \bar{U}_{i 2}^{c d}=\tilde{U}^{c d}, \quad \operatorname{Tr} \rho \bar{V}_{i p}=\operatorname{Tr} \rho \bar{J}_{i}^{ \pm}=0$ and the averaged Hamiltonian $\bar{H}_{F}$ is reduced to Hamiltonian (2.6).

\section{References}

1. Manske, D.: Theory of unconventional superconductors. SpringerVerlag Berlin Heidelberg, New York (2004)

2. Bussmann-Holder, A.: Keller H. in Polarons in advanced materials, edited by A.S. Alexandrov (AA Dordrecht, The Netherlands, 2007) and refs. therein.

3. Robaszkiewicz, S., Micnas, R., Ranninger, J.: Phys. Rev. B 36 , 180 (1987)

4. K.I. Wysokiński in New Developments in High Temperature Superconductivity, edited by J. Klamut, B.W. Veal, B.M. Dabrowski, P.W. Klamut, M. Kazimierski, (Springer-Verlag Berlin Heidelberg New York, 2000)

5. Micnas, R., Ranninger, J., Robaszkiewicz, S.: Rev. Mod. Phys 62, 113 (1990)

6. Pawłowski, G., Robaszkiewicz, S.: Acta Phys. Polon. A 94, 683699 (1998)

7. Czart, W.R., Robaszkiewicz, S.: Acta Phys. Polon. A 100, 75-88 (2001)

8. Czart, W.R., Robaszkiewicz, S.: Int. J. of Mod. Phys. B 15, 3125 $3142(2001)$

9. Emery, V.J.: Phys. Rev. Lett. 58, 2794 (1987)

10. Yu, C.C., Anderson, P.W.: Phys. Rev. B 29, 6165 (1984)

11. Dias da Silva, L.G.G., Dagotto, E.: Phys. Rev. B 79, 155302 (2009)

12. Yashiki, S., Ueda, K.: J. Phys. Soc. Jpn. 80, 084717 (2011)

13. Yashiki, S., Kirino, S., Ueda, K.: J. Phys. Soc. Jpn. 79, 093707 (2010)

14. Hattori, K.: Phys. Rev. B 85, 214411 (2012)

15. Hotta, T., Ueda, K.: Phys. Rev. Lett. 108, 247214 (2012) 
16. Hattori, K.: J. Phys. Soc. Jpn 82, 064709 (2013)

17. Lavagna, M., Pépin, C.: Acta Phys. Polon. B 29, 3753 (1998)

18. Hewson, A.C.: The Kondo Problem to Heavy Fermions. Cambridge Universiy Press, Cambridge (1997)

19. Gusmao, M.A., Aligia, A.A. cond-mat.str-el (2000)

20. Schrieffer, J.R., Wolff, P.A.: Phys. Rev. 149, 491 (1966)

21. Moskalenko, V.A.: FMM 8, 503 (1959)
22. Suhl, H., Mathias, B.T., Walker, L.R.: Phys. Rev. Lett. 3, 532 (1959)

23. Czerwonko, J.: Physica C 235-240, 2337 (1994)

24. Czerwonko, J.: Phys. Mol. Rep. 12, 79 (1995)

25. Wiethege, W., Entel, P., Mühlschlegel, B.Z.: Phys. B Condens. Matter 47, 35-44 (1982)

26. Tarasewicz, P.: J. Supercond. Nov. Magn. 25, 363-375 (2012) 